\title{
Biowaste-derived porous carbon with tuned microstructure for high-energy quasi-solid-state supercapacitors
}

Zhiwei Li, ${ }^{a, *}$ Zhengyu Bai, ${ }^{b, *}$ Hongyu Mi, ${ }^{a *}$ Chenchen Ji, ${ }^{a}$ Song Gao, ${ }^{a}$ and Huan Pang, ${ }^{c *}$

${ }^{\text {a }}$ School of Chemistry and Chemical Engineering, Xinjiang University, 666 Shengli Road, Urumqi 830046, China

${ }^{\mathrm{b}}$ School of Chemistry and Chemical Engineering, Key Laboratory of Green Chemical Media and Reactions, Ministry of Education, Henan Normal University, 46 East of Construction Road, Xinxiang 453007, China

${ }^{\mathrm{c}}$ School of Chemistry and Chemical Engineering, Yangzhou University, 88 Daxue South Road, Yangzhou 225009, China

$\$$ The authors contributed equally.

* Corresponding authors: mmihongyu@163.com (H.M.); panghuan@yzu.edu.cn (H.P.).

Number of pages: 7

Number of Figures: 6

Number of Tables: 1 


\section{Experimental}

\section{Regents and Materials}

Sodium sulfate $\left(\mathrm{Na}_{2} \mathrm{SO}_{4}\right)$, ethanol $\left(\mathrm{C}_{2} \mathrm{H}_{5} \mathrm{OH}\right)$ and potassium hydroxide $(\mathrm{KOH})$ were analytical reagents. Polyvinyl alcohol (PVA, $\left.\mathrm{M}_{\mathrm{w}} \approx 95000\right)$ was received from Beijing J\&K Scientific Co., Ltd (China). Carboxymethylcellulose sodium (CMC-Na, viscosity: $\geq 1900 \mathrm{mPa} \cdot \mathrm{s}$ ) was purchased from Tokyo Chemical Industry Co., Ltd (Japan). A commercial activated carbon (YP-50F) was purchased from Kuraray Co., Ltd (Japan), and Soybean dregs (SDs) were obtained by a soybean-milk retail store.

\section{Material Preparation}

In a typical run, $3 \mathrm{~g}$ of clean SDs were dispersed into $80 \mathrm{~mL}$ of a $\mathrm{KOH}$ solution. The slurry was subsequently subjected to a hydrothermal process under $100{ }^{\circ} \mathrm{C}$ for $6 \mathrm{~h}$. The mixture after centrifuging and drying was heated in a tube furnace from room temperature to $650{ }^{\circ} \mathrm{C}$ under a $\mathrm{N}_{2}$ flow with a heating rate of $5{ }^{\circ} \mathrm{C} \min ^{-1}$, and remained at this temperature for $2 \mathrm{~h}$. Finally, the mixture obtained was washed thoroughly with $1.0 \mathrm{M} \mathrm{HCl}$ solution and deionized water, and dried overnight. For convenience, final carbons were labeled as $\mathrm{HSC}-\mathrm{x}$, where $\mathrm{x}$ represents the concentration of $\mathrm{KOH}$ solution. The carbon yields of HSC-0.00, HSC-0.05, HSC-0.25, HSC-0.50 and HSC-1.00 are 12.3, 10.2, 8.0, 6.4 and $3.2 \mathrm{wt} \%$, respectively.

\section{Physical Characterizations}

Field-emission scanning electron microscopy (FE-SEM) and energy dispersive X-ray spectroscopy (EDS) analyses were performed with a Hitachi SU-8010. Transmission electron microscopy (TEM) and high-resolution transmission electron microscopy (HR-TEM) measurements were conducted on a Hitachi-600 and a JEM-2100F, respectively. X-ray diffraction (XRD) was recorded on a Brüker D8 Advance X-ray diffractometer. Raman spectrum was obtained from a Brüker Senterra 
Raman spectrometer. X-ray photoelectron spectroscopy (XPS) spectrum was provided by an ESCALAB 250Xi spectrometer. Element analysis was carried out by Vario EL cube organic element analyzer. The $\mathrm{N}_{2}$ sorption isotherm was tested at $77 \mathrm{~K}$ using a 3H-2000PM1/2 sorptometer. The specific surface area (SSA) was calculated by the Brunauer-Emmentt-Teller (BET) method and pore size distribution (PSD) was estimated by using the density functional theory (DFT) method.

\section{Electrochemical measurements}

To study the electrochemical profile of the products including cyclic voltammetry (CV), galvanostatic charge/discharge (GCD), electrochemical impedance spectroscopy (EIS) and cyclic stability, a typical three-electrode cell was fabricated with the working electrode (WE), platinum counter electrode and $\mathrm{Hg} / \mathrm{HgO}$ reference electrode in a $\mathrm{KOH}$ electrolyte. The WE was prepared according to our previous report [1]. In detail, the uniform mixture of the as-prepared activated carbon, acetylene black and polytetrafluoroethylene with a mass ratio of 8:1:1 was firstly rolled into a thin sheet, and then the cut small sheet (diameter: $1 \mathrm{~cm}$ ) was compressed on current collector (nickel foam for alkaline case and graphite paper for neutral case, respectively) as the working electrode, in which the loading of active material was $2.5-20 \mathrm{mg} \mathrm{cm}^{-2}$. Gravimetric capacitance $\left(C_{\mathrm{g}}, \mathrm{F} \mathrm{g} \mathrm{g}^{-1}\right)$, volumetric capacitance $\left(C_{\mathrm{v}}, \mathrm{F}\right.$ $\left.\mathrm{cm}^{-3}\right)$ and areal capacitance $\left(C_{\mathrm{a}}, \mathrm{F} \mathrm{cm} \mathrm{cm}^{-2}\right)$ of the electrode were defined using eqns $(1-3)[2]:$

$C_{\mathrm{g}}=\frac{I \Delta t}{m \Delta V}$

$C_{\mathrm{v}}=C_{\mathrm{g}} \times \rho$

$C_{\mathrm{a}}=C_{\mathrm{g}} \times m$

where $I(\mathrm{~A}), \Delta t(\mathrm{~s}), m(\mathrm{~g}), \Delta V(\mathrm{~V})$ and $\rho\left(\mathrm{g} \mathrm{cm}^{-3}\right)$ refer to the discharge current, the discharge time, the mass of active material, the potential window after ohmic drop for 
the working electrode and the packing density of active material.

For QSSCs, two same WEs of the HSC-0.50 (active material loading on the single electrode: $5 \mathrm{mg}$ ) were stacked together with a PVA/KOH electrolyte in between. Generally, the PVA/KOH electrolyte was prepared by dissolving PVA (1 g) and $\mathrm{KOH}$ (1 g) in $10 \mathrm{~mL}$ deionized (DI) water under vigorous stirring at $85{ }^{\circ} \mathrm{C}$, and further being settled about $12 \mathrm{~h}$ to form an ivory-white film for use. The YP-50F based QSSCs were also assembled and investigated for comparison. To further improve the energy density of QSSCs, we built a neutral SSC face-to-face with two identical HSC-0.50 electrodes and $\mathrm{CMC}-\mathrm{Na} / \mathrm{Na}_{2} \mathrm{SO}_{4}$ gel electrolyte, in which a Whatman filter was used as separator. The neutral gel was prepared via facile mixing of $0.2 \mathrm{~g}$ CMC-Na powder and $10 \mathrm{~mL} \mathrm{Na} \mathrm{SO}_{4}$ solution $\left(0.25 \mathrm{~mol} \mathrm{~L}^{-1}\right)$ until a clear solution formed under room temperature. The gravimetric capacitance $\left(C_{\mathrm{sg}}, \mathrm{F} \mathrm{g} \mathrm{g}^{-1}\right)$ and volumetric capacitance $\left(C_{\mathrm{sv}}, \mathrm{F} \mathrm{cm}^{-3}\right)$ of single electrode in SSCs was determined using eqns (4) and (5) [2]:

$C_{\mathrm{sg}}=2 \frac{I \Delta t}{m \Delta V}$

$C_{\mathrm{sv}}=C_{\mathrm{sg}} \times \rho$

where $m(\mathrm{~g})$ and $\Delta V(\mathrm{~V})$ are the loading of active material in one electrode and the voltage window excluding ohmic drop for the solid-state device.

The energy density $\left(E, \mathrm{Wh} \mathrm{kg}^{-1}\right)$ and power density $\left(P, \mathrm{~W} \mathrm{~kg}^{-1}\right)$ of the device were calculated by eqns (6) and (7) $[1,4]$ :

$$
\begin{aligned}
& E=\frac{1}{2 \times 4 \times 3.6} C_{\mathrm{sg}} \Delta V^{2} \\
& P=3600 \frac{E}{\Delta t}
\end{aligned}
$$




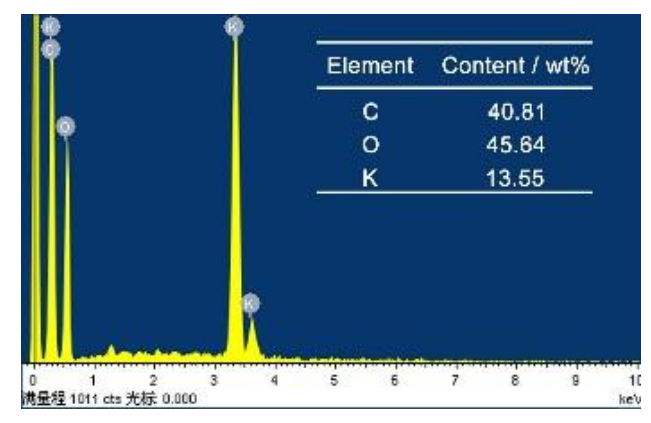

Figure S1. EDS spectrum of HSK-0.50.
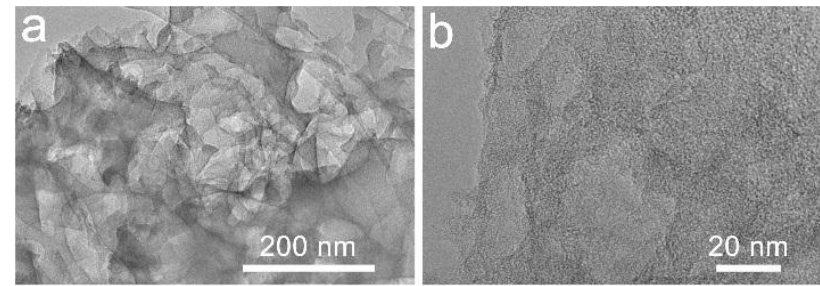

Figure S2. HR-TEM images of HSC-0.50.
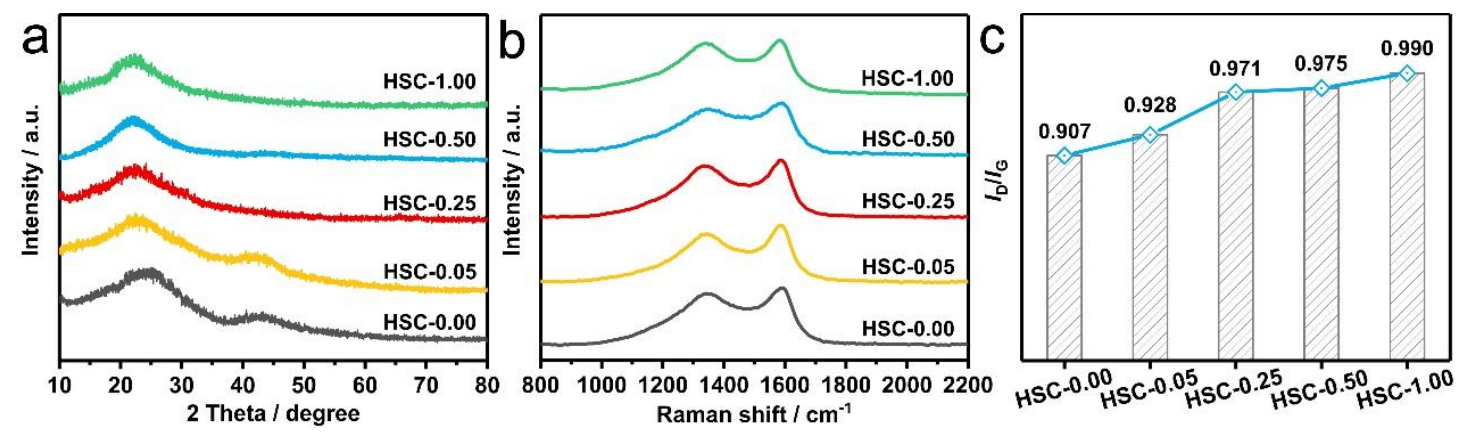

Figure S3. (a) XRD patterns, (b) Raman spectra, and (c) $I_{\mathrm{D}} / I_{\mathrm{G}}$ values obtained from Raman results.

Table S1 Chemical compositions of various HSC-x obtained from element analysis

\begin{tabular}{ccccc}
\hline Sample & $\mathrm{C}(\mathrm{wt} \%)$ & $\mathrm{H}(\mathrm{wt} \%)$ & $\mathrm{N}(\mathrm{wt} \%)$ & $\mathrm{O}(\mathrm{wt} \%)$ \\
\hline HSC-0.00 & 78.6 & 2.1 & 5.7 & 13.6 \\
HSC-0.05 & 82.2 & 3.6 & 2.5 & 11.7 \\
HSC-0.25 & 83.7 & 3.8 & 1.8 & 10.7 \\
HSC-0.50 & 86.2 & 4.8 & 1.2 & 7.8 \\
HSC-1.00 & 86.7 & 5.6 & 0.6 & 7.1 \\
\hline
\end{tabular}



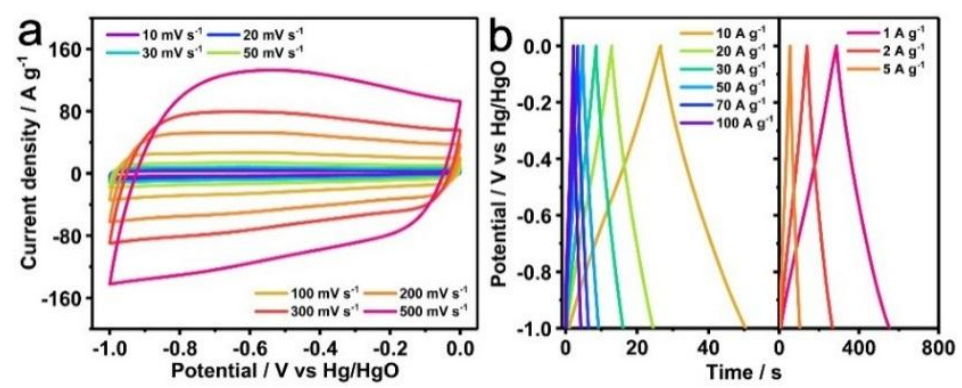

Figure S4. (a) CV curves at various scan rates and (b) GCD curves at various current densities for HSC-0.50 electrode with 6.0 M KOH electrolyte.
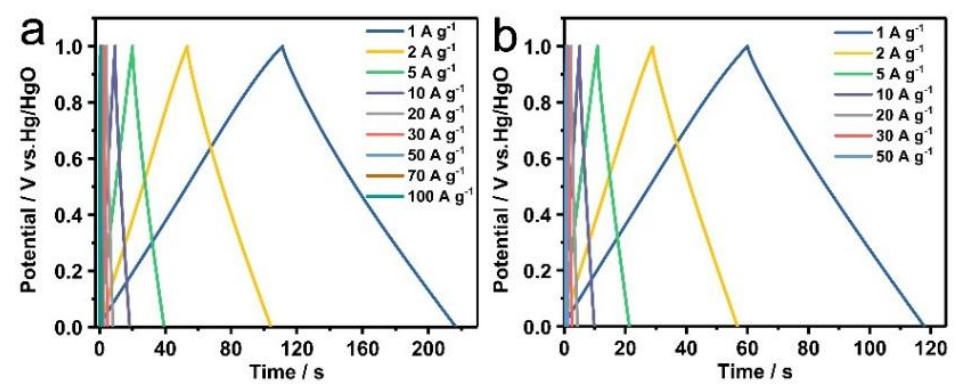

Figure S5. GCD curves at various current densities for (a) HSC-0.50 and (b) YP-50F QSSCs with PVA/KOH gel electrolyte.

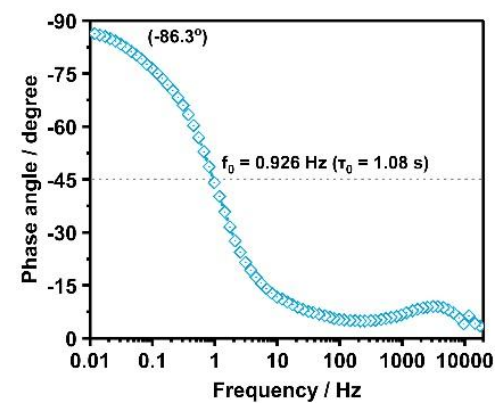

Figure S6. Bode phase diagram for HSC-0.50 QSSC with PVA/KOH gel electrolyte. 


\section{References}

(1) Li, Z.; Chen, N.; Mi, H.; Ma, J.; Xie, Y.; Qiu, J. Hierarchical hybrids integrated by dual polypyrrole-based porous carbons for enhanced capacitive performance. Chem. Eur. J., 2017, $23,13474-13481$.

(2) Liu, M.; Niu, J.; Zhang, Z.; Dou, M.; Wang, F. Potassium compound-assistant synthesis of multi-heteroatom doped ultrathin porous carbon nanosheets for high performance supercapacitors. Nano Energy, 2018, 51, 366-372.

(3) Sheng, L.; Chang, J.; Jiang, L.; Jiang, Z.; Liu, Z.; Wei, T.; Fan, Z. Multilayer-folded graphene ribbon film with ultrahigh areal capacitance and high rate performance for compressible supercapacitors. Adv. Funct. Mater., 2018, 28, 1800597-1800606.

(4) Li, Z.; Mi, H.; Liu, L.; Bai, Z.; Zhang, J.; Zhang, Q.; Qiu, J. Nano-sized ZIF-8 anchored polyelectrolyte-decorated silica for nitrogen-rich hollow carbon shell frameworks toward alkaline and neutral supercapacitors. Carbon, 2018, 136, 176-186. 